\title{
Magnetic Moments of Excited Baryons
}

\author{
Volker Metag ${ }^{1, \star}$ \\ ${ }^{1}$ II. Physikalisches Institut, Universität Gießen, Heinrich Buff Ring 16, 35392 Gießen
}

\begin{abstract}
In project A.3, the reaction $\gamma p \rightarrow \pi^{0} \gamma^{\prime} p$ has been studied using the TAPS photon spectrometer in the energy range $\sqrt{s}=1221-1331 \mathrm{MeV}$. Energy tagged photon beams have been produced with the Glasgow tagging spectrometer from electron beams provided by the MAMI-B accelerator. Angle and energy differential cross sections have been measured and compared to theoretical calculations. This comparison allows the magnetic moment of the $\Delta^{+}$isobar to be extracted for the first time to $\mu_{\Delta^{+}}=\left[2.7_{-1.0}^{+1.3}(\mathrm{stat}) \pm 1.5(\mathrm{syst}) \pm 3\right.$ (theo) $] \mu_{N}$. In an extension of the A3 project to the meson sector, the time-like transition form factor of the $\eta$ meson has been measured with the Crystal Ball/TAPS detector system at MAMI-C.
\end{abstract}

\section{Introduction}

The main goal of the Transregio TR16 is to explore the excitation energy spectrum of the nucleon in order to unravel its complex structure. Apart from determining the mass, width and quantum number of excited states, information on the polarizability, magnetic moments and form factors provide further insight in the structure of the nucleon. For baryon ground states these properties have been measured with great precision. Corresponding information on excited states has so far been lacking. Because of its prominent position in the excitation energy spectrum, the properties of the $\Delta(1232)$ resonance are of particular importance.

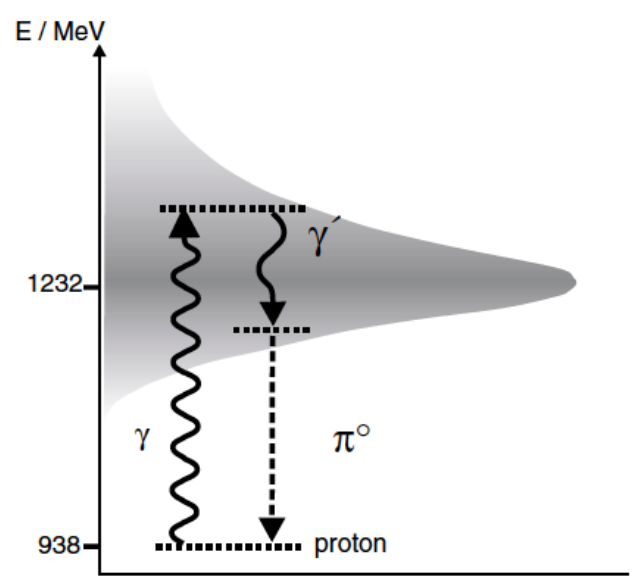

Figure 1. Scheme for studying the static electromagnetic properties of the $\Delta(1232)$ isobar. The $\gamma^{\prime}$ transition carries the information on the magnetic moment of the $\Delta^{+}$.
Following pioneering experiments determining the magnetic moment of the $\Delta^{++}$resonance $[1,2]$, a first attempt has been made within the A3 project to measure the magnetic moment of the $\Delta^{+}$resonance. The idea of the experiment is sketched in Fig. 1. The broad $\Delta^{+}$resonance $(\Gamma=120 \mathrm{MeV})$ is populated in the high mass regime via photon excitation, followed by a $\gamma$ transition within the resonance and a subsequent $\pi^{0}$ decay of the $\Delta^{+}$resonance to the nucleon ground state. Spin and parity conservation require that - to lowest multipole order - this $\gamma^{\prime}$ transition is of magnetic dipole (M1) type. The next allowed multipole is electric quadrupole (E2), but the E2/M1ratio of the $\Delta-\mathrm{N}$ transition has been measured to be small $(0.025)$ [3], indicating a small quadrupole deformation of the $\Delta^{+}$ resonance. The magnetic octupole transition (M3) is suppressed by two additional powers of the photon energy. Thus, the measurement of the $\gamma p \rightarrow \pi^{0} \gamma^{\prime} p$ reaction provides access to $\mu_{\Delta^{+}}$. The sensitivity of the $\Delta^{+} \rightarrow \Delta^{+} \gamma^{\prime}$ amplitude to the magnetic moment $\mu_{\Delta^{+}}$has been investigated theoretically by Machavariani et al. [4, 5] and Drechsel [6]. The same $\pi^{0} \gamma^{\prime} p$ final state can, however, also result from bremsstrahlung radiation of the $\Delta^{+}$or the proton. For a comparison with the experiment, these channels have to be included in a calculation as in [7]. Fig. 2 shows the graph of interest (a2) and all other graphs contributing to the $\gamma p \rightarrow \pi^{0} \gamma^{\prime} p$ reaction. Our experimental data will be compared to these extended calculations by Drechsel and Vanderhaegen [7].

\section{The experiment}

The reaction $\gamma p \rightarrow \pi^{0} \gamma^{\prime} p$ was measured at the electron accelerator MAMI-B, using the Glasgow Tagged photon facility [8] and the photon spectrometer TAPS [9, 10]. A quasi monochromatic photon beam was produced in the energy range of 205-820 MeV via bremsstrahlung tagging. The 510 hexagonal $\mathrm{BaF}_{2}$ TAPS modules were arranged in

\footnotetext{
^e-mail: Volker.Metag@exp2.physik.uni.giessen.de
} 

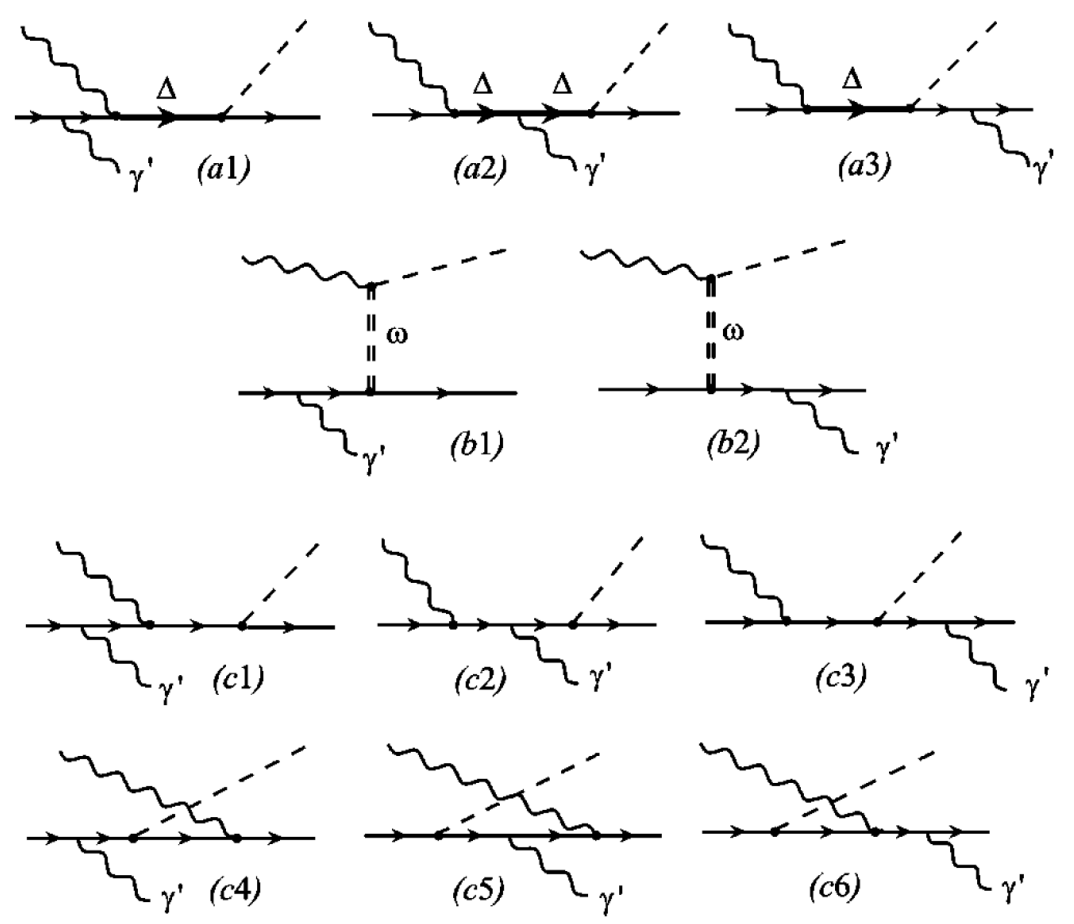

Figure 2. Diagrams considered in the calculation of the $\gamma p \rightarrow \pi^{0} \gamma^{\prime} p$ reaction in the $\Delta(1232)$ energy region [7].

a horizontal plane around the liquid hydrogen target in 6 blocks of 62 detectors and a forward wall of 138 detectors. The setup covered about $40 \%$ of the full solid angle. Further details of the experimental setup and the running conditions can be found in [11].

\section{Data analysis}

The $\gamma p \rightarrow \pi^{0} \gamma^{\prime} p$ was measured exclusively since the 4momentum vectors of all final state particles were determined. The $\pi^{0}$ mesons were detected via their two photon decay and identified via an invariant mass analysis. The third photon in the final state was assigned to the $\gamma$ ' transition. The protons were identified by time-of-flight, exploiting the excellent time resolution of TAPS, and by the energy deposited in the $\mathrm{BaF}_{2}$ crystals. The main experimental background was the $2 \pi^{0}$ channel where one of the 4 decay photons escaped undetected. This background could be removed by a cut in the $\pi^{0} p$ missing mass spectrum shown in Fig. 3. For the $\gamma p \rightarrow \pi^{0} \gamma^{\prime} p$ reaction the missing mass is 0 , while $2 \pi^{0}$ events with one decay photon lost were centered around a missing mass of $0.02 \mathrm{GeV}^{2}$.

\section{Results}

The measured angle and energy differential cross sections are shown in Fig. 4. For the higher incident photon energies a clear deviation from a $1 / \mathrm{E}_{\gamma}$ bremsstrahlung distribution is observed, indicating contributions from $\gamma^{\prime}$ transitions within the $\Delta^{+}$resonance. The data are compared with calculations by Drechsel and Vanderhaegen [7] for
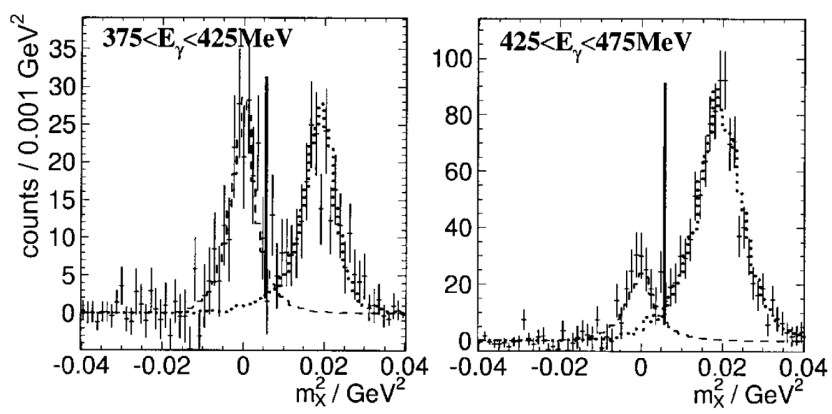

Figure 3. Missing mass of the $\pi^{0} p$ system for two incident photon energy bins. The peak near $0.02 \mathrm{GeV}^{2}$ originates from $2 \pi^{0}$ production and is cut away. The peak at $0 \mathrm{GeV}^{2}$ corresponds to the $\pi^{0} \gamma^{\prime} p$ exit channel. The dashed and dotted curves show the corresponding line shapes simulated with GEANT3.

different values of $\kappa_{\Delta^{+}}$, related to the magnetic moment of the $\Delta^{+}$resonance by

$$
\mu_{\Delta^{+}}=\left(1+\kappa_{\Delta^{+}}\right) \frac{m_{N}}{m_{\Delta}} \mu_{N}
$$

The calculations follow the overall shape of the experimental distributions but overestimate the data for the highest excitation energy. A model independent determination of the $\gamma p \rightarrow \pi^{0} \gamma^{\prime} p$ cross section is possible in the soft photon limit, which relates the $\pi^{0} \gamma^{\prime} p$ production to the 

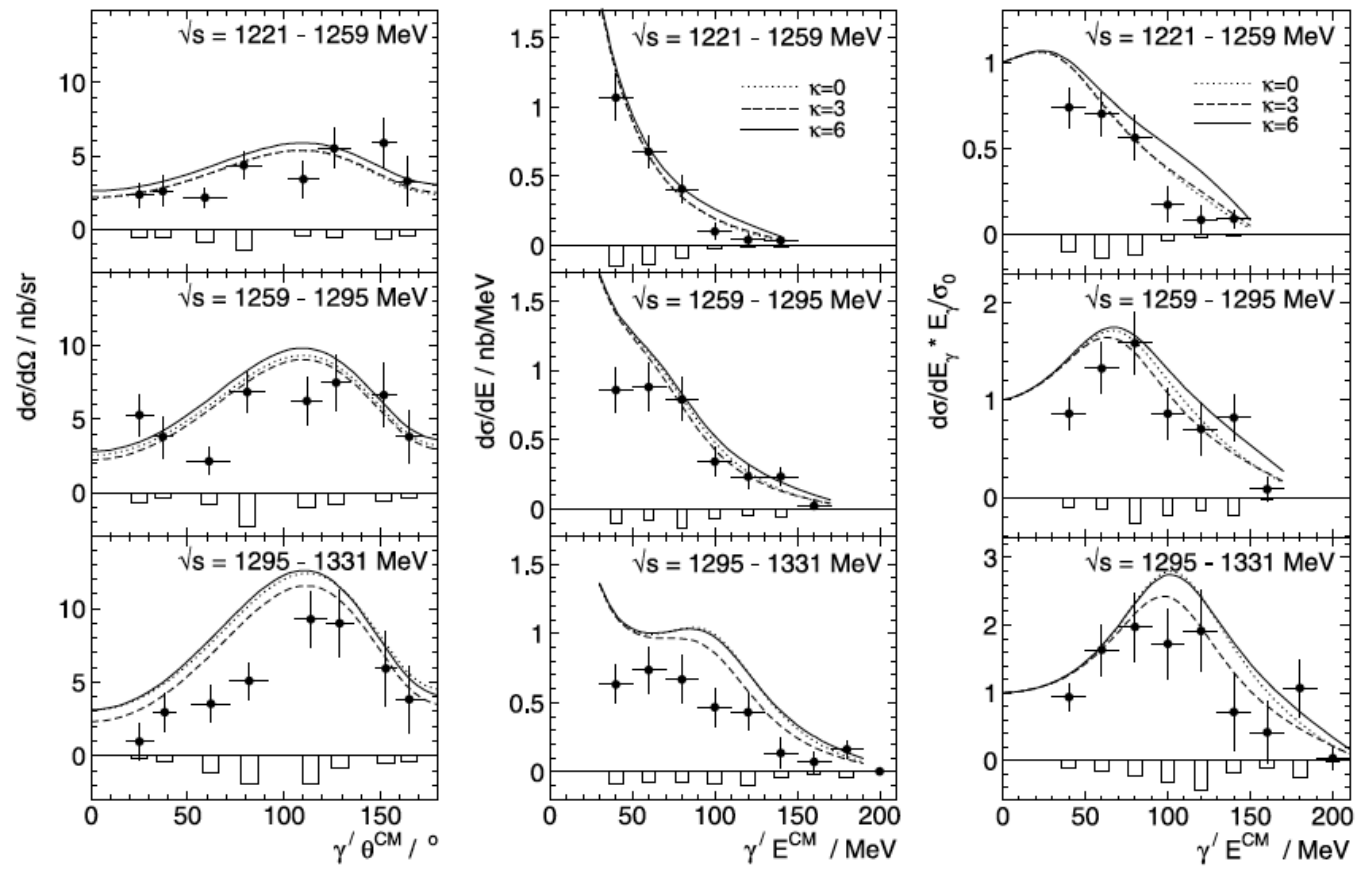

Figure 4. Differential cross sections for three excitation energy bins $\sqrt{s}$ in the center of mass system. The curves represent calculations [7] for $\kappa_{\Delta^{+}}=0,3$, and 6. Bars indicate the systematic errors. (Left) Angular distribution of the $\gamma^{\prime}$ photon. (Middle) Energy distribution of the $\gamma^{\prime}$ photon. (Right) Energy distribution divided by the soft photon limit $\frac{\sigma_{0}}{E_{\gamma}}$ for data and calculations.

$\pi^{0} p$ production in the limit of vanishing photon energy $E_{\gamma^{\prime}}$. The ratio of both cross sections approaches unity for small transition energies $E_{\gamma^{\prime}}$ (see Fig. 4). The cross section ratios are less sensitive to experimental and theoretical uncertainties and are thus used to extract $\mu_{\Delta^{+}}$. A combined maximum likelihood analysis of all three cross section ratios in Fig. 4 (right column) gives a magnetic moment of the $\Delta^{+}$ resonance of $\mu_{\Delta^{+}}=\left[2.7_{-1.0}^{+1.3}\right.$ (stat) \pm 1.5 (syst) \pm 3 (theo) $] \mu_{N}$; the goodness of the fit is $\chi^{2} / f=1.8(f=21)$. The first error represents the statistical uncertainty, and the second one reflects the systematic errors of the experiment indicated in Fig. 4. The systematic uncertainty of the model calculation is estimated to be $\approx \pm 3 \mu_{N}$. This determination of $\mu_{\Delta^{+}}$is unfortunately not precise enough to allow a detailed test of different baryon structure calculations.

The above analysis is simplified in so far as the magnetic moment of the $\Delta$ - as of any other unstable state - is a complex quantity. Treating the $\Delta$ as a resonance (not as a stable particle), an explicit prescription has been developed in project A6 how to extract the $\Delta N \gamma *$ transition form factor from lattice data [12].

In continuation of the A3 project a follow-up experiment was performed at MAMI-B employing the Crystal Ball/TAPS detector system with almost full coverage of the solid angle. The improved experimental conditions led to 60 times higher statistics. The results of these measurements were published in [13]. Despite of the much better data set an improved value of the magnetic moment $\mu_{\Delta^{+}}$could not be extracted because the available calculations did not reproduce in detail the measured high statistics $\gamma^{\prime}$ angle and energy distributions.

\section{The time-like transition form factor of the $\eta$ meson}

In an extension of project $\mathrm{A} 3$ to the meson sector the time-like transition form factor of the $\eta$ meson has been measured using the Crystal Ball/TAPS detector system at MAMI-C. Electromagnetic transition form factors are an important tool for studying the intrinsic structure of hadrons. The decay of light pseudoscalar mesons $\pi^{0}, \eta$, and $\eta^{\prime}$ as well as vector mesons $\rho, \omega$ and $\phi$ allow a test of the vector meson dominance (VMD) conjecture where the coupling of a photon to a hadron is mediated by a virtual vector meson. For point-like particles the decay rates can be exactly calculated within Quantum Electrodynamics (QED). Information on the intrinsic structure of mesons is encoded in the multiplicative transition form factor. The transition form factors can be studied for momentum transfers not accessible in annihilation or scattering experiments. Furthermore, new theoretical efforts aiming at a systematic description of light pseudoscalar and vector mesons and their couplings to electromagnetism [14] can be tested. An overview over earlier work in this field can be found in [15].

In this experiment the $\eta$ Dalitz decay $\eta \rightarrow \gamma \gamma * \rightarrow \gamma$ $\mathrm{e}^{+} \mathrm{e}^{-}$with a branching ratio of $6 \cdot 10^{-3}$ has been investigated. The reconstruction of such a weak decay channel is a major experimental challenge and could only succeed 

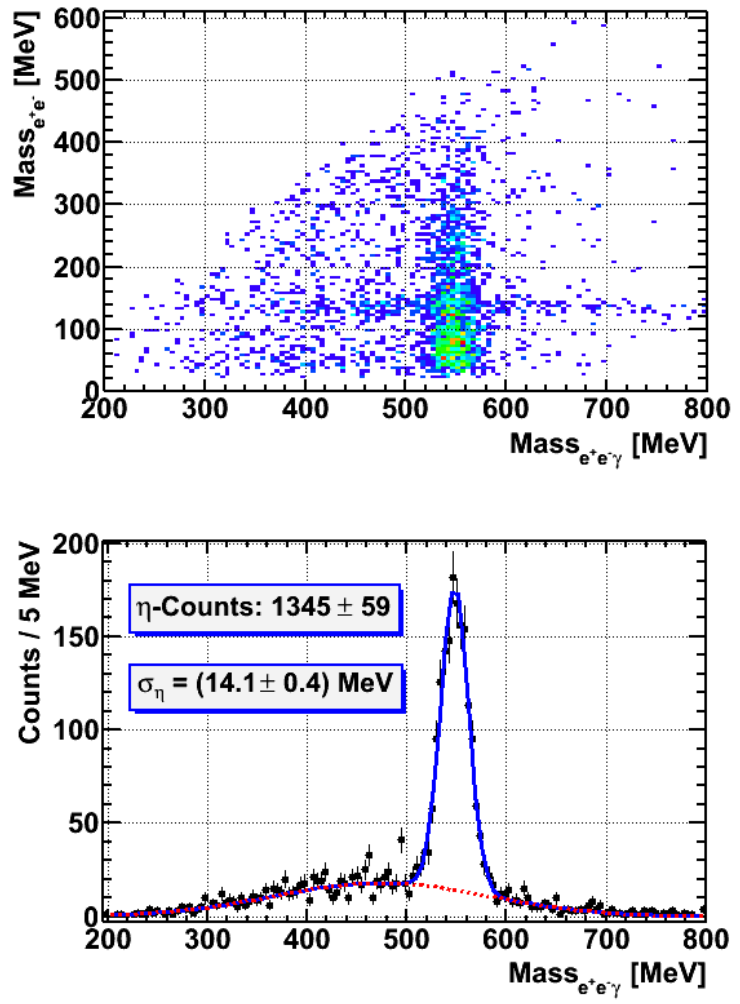

Figure 5. Distribution of reconstructed $\mathrm{e}^{+} \mathrm{e}^{-} \gamma$ candidate events after applying the selection cuts. The lower figure shows the projection onto the $\mathrm{e}^{+} \mathrm{e}^{-} \gamma$ invariant mass axis [16].

by using the almost complete $4 \pi$ coverage of the combined Crystal Ball and TAPS detector system at MAMI-C. The disadvantage of the detector system is that it does not provide a magnetic field for charged particle tracking. Only the energy and angle of registered particles is available and the information whether they are charged or neutral. The sign of the charge cannot be determined.

The identification of this weak decay channel required a series of cuts described in detail in [16]. These cuts reduced the reconstruction efficiency of the $\gamma p \rightarrow p \eta \rightarrow$ $p \gamma \mathrm{e}^{+} \mathrm{e}^{-}$reaction to about $5 \%$. For events that have survived these cuts the correlation between the $\mathrm{e}^{+} \mathrm{e}^{-}$invariant mass and the $\mathrm{e}^{+} \mathrm{e}^{-} \gamma$ invariant mass as well as the projection on the $\mathrm{e}^{+} \mathrm{e}^{-} \gamma$ invariant mass axis are shown in Fig. 5. A total of $1345 \eta \rightarrow \gamma \mathrm{e}^{+} \mathrm{e}^{-}$events residing on a small background could be reconstructed. Fitting the $\gamma \mathrm{e}^{+} \mathrm{e}^{-}$mass spectra for different slices in Mass $_{e^{+} e^{-}}$, the Mass $e_{e^{+} e^{-}}$distribution shown in Fig. 6 is obtained. The mass distribution is compared to the QED prediction for a point-like meson. Dividing the measured Mass $_{e^{+} e^{-}}$distribution by the QED prediction, the time-like transition form factor of the $\eta$ meson is obtained as shown in Fig. 7. Fitting the form factor using the standard VMD parametrization

$$
F\left(m^{2}\right)=\frac{1}{1-(m / \Lambda)^{2}},
$$

a value of $\Lambda^{-2}=(1.92 \pm 0.35$ (stat) \pm 0.13 (syst) $) \mathrm{GeV}^{-2}$ is obtained corresponding to $\Lambda=(720 \pm 60$ (stat $) \pm 25$

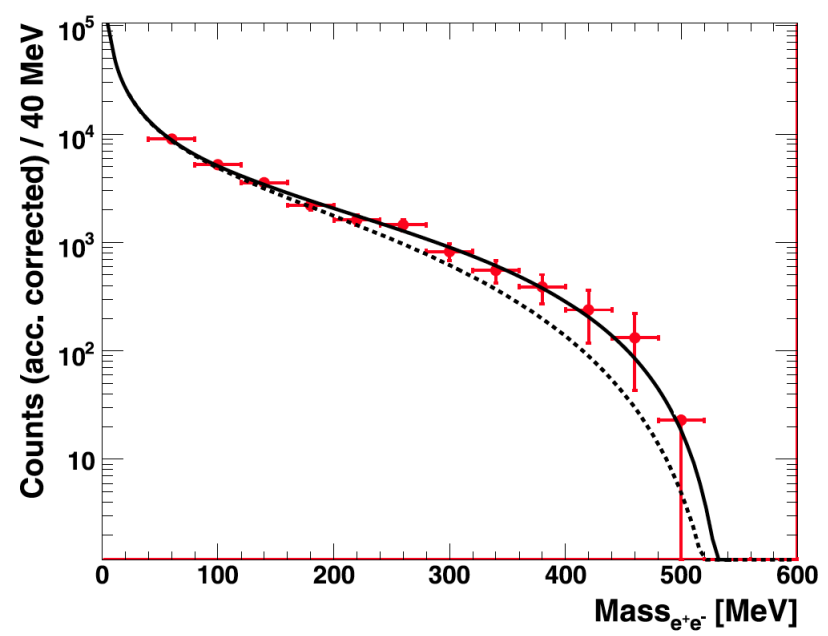

Figure 6. Invariant $\mathrm{e}^{+} \mathrm{e}^{-}$mass distribution of reconstructed events after acceptance correction [16].The errors given are purely statistical.The solid curve is a fit within the VMD-model, using a monopole form factor (Eq. 2). The dotted curve is the QED prediction.

(syst)) MeV. The systematic error is estimated from a comparison of results obtained for different cut settings. The statistics is improved by an order of magnitude compared to the most recent form factor measurement in the $\mathrm{e}^{+} \mathrm{e}^{-}$channel [17]. The data are in good agreement with the high statistics NA60 data, obtained in the $\mu^{+} \mu^{-}$ channel, however, without a full reconstruction of the $\eta$ meson, and with recent theoretical calculations [19]. After establishing in this pioneering experiment that weak

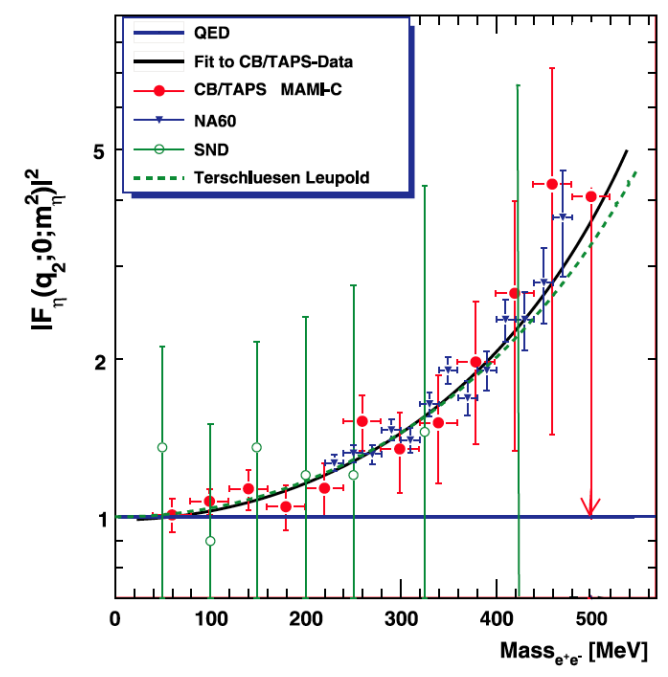

Figure 7. Dalitz transition form factor. The red circles are the data of this work and the black curve is a fit to these data [16]. The green (open) circles show the result of the SND experiment [17]. The blue (inverted) triangles represent the results obtained by the NA60 collaboration in the $\mu^{+} \mu^{-}$channel [18]. The green (dashed) curve is a calculation performed by [19].

decay channels like the $\eta$ Dalitz decay can be identified in an exclusive analysis using a highly segmented $4 \pi$ 
calorimeter without magnetic field, a series of measurements has been performed within the A2 collaboration at MAMI. An improved measurement of the $\eta$ Dalitz decay has been reported in [20]. Including all runs on a liquid hydrogen target and applying kinematic fitting routines a large data set has been accumulated for further improving the $\eta$ form factor determination and to extend the analysis to the $\omega \rightarrow \pi^{0} \mathrm{e}^{+} \mathrm{e}^{-}$Dalitz decay [21]. On the theoretical side, improved calculations based on dispersion theory have been performed within project B3 for the $\eta, \omega$ and $\phi$ transition form factors $[22,23]$.

I would like to thank all the colleagues in the A2 collaboration at MAMI for their support and contributions to the results described in this article. The results are based on the theses of the strongly engaged $\mathrm{PhD}$-students $\mathrm{M}$. Kotulla and $\mathrm{H}$. Berghäuser. Illuminating discussions with D. Drechsel, V. Pascalutsa and M. Vanderhagen on the theoretical interpretation of the data are highly appreciated. The work reported here would not have been possible without the support from the Deutsche Forschungsgemeinschaft within the SFB/TR16.

\section{References}

[1] B. Nefkens et al., Phys. Rev. D 18, 3911 (1978).

[2] A. Bosshard et al., Phys. Rev. D 44, 930 (1991).

[3] R. Beck et al., Phys. Rev. Lett. 78, 606 (1997).

[4] A. Machavariani, A. Faessler and A. Buchmann, Nucl. Phys. A 646, 231 (1999).
[5] A. Machavariani, A. Faessler and A. Buchmann, Nucl. Phys. A 686, 601 (2002).

[6] D. Drechsel, Phys. Lett. B 484, 236 (2000).

[7] D. Drechsel and M. Vanderhaegen, Phys. Rev. C 64, 065202 (2001).

[8] S. Hall et al., Nucl. Inst. Methods Phys. Res. A 368, 698 (1996).

[9] R. Novotny, et al., IEEE Trans. Nucl. Sci 38, 392 (1991).

[10] A. R. Gabler et al., Nucl. Instr. Methods Phys. Res. A 346, 168 (1994).

[11] M. Kotulla et al., Phys. Rev. Lett. 89, 278001 (2002).

[12] A. Agadjanov et al., Nucl. Phys. B 886, 1199 (2014).

[13] S. Schuman et al., Eur. Phys. J. A 43, 269 (2010).

[14] M. F. M. Lutz and S. Leupold, Nucl. Phys. A 813, 96 (2008).

[15] L. G. Landsberg, Phys. Rep. 128, 301 (1985).

[16] H. Berghäuser et al., Phys. Lett. B 701, 562 (2011).

[17] M. N. Achasov et al., Phys. Lett. B 504, 275 (2001).

[18] R. Arnaldi et al., Phys. Lett. B 677, 260 (2009).

[19] C. Terschlüsen, Diploma thesis, Univ. of Giessen, 2010.

[20] P. Aguar-Bartolome et al., Phys. Rev. C 89, 044608 (2014).

[21] S. Prakhov, private communication (2016).

[22] C. W. Xiao et al., arXiv:1509.02194.

[23] S. P. Schneider et al., Phys. Rev. D 86054013 (2012). 